\title{
SARS-CoV-2: Enhancement and Segmentation of High-ResolutionMicroscopy Images. Part I
}

Roberto Rodriguez ( $\nabla$ rrm@icimaf.cu )

ICIMAF

\section{Brian A. Mondeja}

CEA

\section{Odalys Valdes}

IPK

\section{Sonia Resik}

IPK

Ananayla Vizcaino

CEA

Emilio F. Acosta

CEA

Yorexis González

CEA

Vivian Kourí

IPK

Angelina Díaz

CEA

María G. Guzmán

IPK

\section{Research Article}

Keywords: Image enhancement, Segmentation, Algorithms, Coronavirus, SARS-CoV-2, Microscopy

Posted Date: August 28th, 2020

DOI: https://doi.org/10.21203/rs.3.rs-65818/v1

License: (9) This work is licensed under a Creative Commons Attribution 4.0 International License. Read Full License 
Version of Record: A version of this preprint was published at Signal, Image and Video Processing on August 28th, 2020. See the published version at https://doi.org/10.1007/s11760-021-01912-7. 


\title{
SARS-CoV-2: Enhancement and Segmentation of High-Resolution Microscopy Images. Part I
}

\author{
Roberto Rodríguez ${ }^{1 *}$, Brian A. Mondeja ${ }^{2}$; Odalys Valdes ${ }^{3}$; Sonia Resik ${ }^{3}$; Ananayla Vizcaino ${ }^{2}$; Emilio F. Acosta ${ }^{2}$; Yorexis \\ González ${ }^{2}$; Vivian Kourî́3 ${ }^{3}$ Angelina Díaz²; María G. Guzmán ${ }^{3}$
}

\begin{abstract}
Possibly, and due to poor eating habits and unhealthy lifestyle, many virus are transmitted to human people. Such is the case, of the novel coronavirus SARS-Cov-2, which has expanded of exponential way, practically, to whole world population. For this reason, the study of real microscopic images of this coronavirus is of great importance. The SARS-Cov-2 images were captured from nasopharyngeal samples of Cuban symptomatic individuals (RT-PCR positives for SARS-CoV-2), and processed via scanning electron microscopy. However, many times these microscopic images present some blurring problems, which are always susceptible to be improved. The aim of this work is to propose new computational methods to carry out enhancement and segmentation of SARS-Cov-2 high-resolution microscopic images. Moreover, due to the importance of the obtained results, this first work will be addressed to the application of the proposed algorithm. The proposed strategy obtained very satisfactory results, and we validated its performance, together with specialist physicians, on a set of 1005 images. A second paper will deeply analyze the theory related to these algorithms.
\end{abstract}

Keywords. Image enhancement, Segmentation, Algorithms, Coronavirus, SARS-CoV-2, Microscopy

\section{Introduction}

Probably, due to poor eating habits and unhealthy lifestyle, many types of viruses are transmitted to humans. For example, this novel virus, named coronavirus due to visual appearance under electron microscopy- similar to a crown [1], it is the cause of an infectious disease by severe acute respiratory syndrome (SARS-Cov-2) [2], and named by the World Health Organization (WHO): COVID-19. This disease has expanded of exponential way, practically, to the whole world population, and already today, it is considered a pandemic.

The fight against COVID-19 has motivated many scientific institutions and researchers from all the specials in the world to seek effective methods and techniques to help end this pandemic. In this direction, the computer vision community has not been left behind, and many articles have been published, mainly for the improvement of Computed Tomography (CT) images using deep learning $[3,4,5,6,7]$.

\footnotetext{
* Corresponding author: Roberto Rodríguez rrm@icimaf.cu

${ }^{1}$ Institute of Cybernetics, Mathematics, and Physics of Cuba (ICIMAF)

${ }^{2}$ Center for Advanced Studies of Cuba (CEA)

${ }^{3}$ Institute of Tropical Medicine "Pedro Kourí “ (IPK)
}

However, it draws attention the small number of published papers for the improvement of microscopic images, when in known the blurring problems inherent in these types of images, which often makes it difficult to analyze correctly them.

In effect, microscopy images, without a doubt, it are widely used for the diagnosis of various diseases such as infections and tumors. These facilitate basic biomedical research that has been continuously generating new knowledges into the causes of human diseases, and for that reason, they are of great importance in improving of quality of health. However, the obtaining high-quality microscopic images is not always a trivial process, even in the best microscopes, due to physical phenomena that originate between the glass coverslip, light and microscope optics. Some of these phenomenons are, scattering, out-of-focus, imperceptible vibrations, voltage disturbances, among others, which causes certain blurring in the microscopic images. Examples of some of them can be seen in $[8,9,10]$.

With the rapid development of computer vision, where its application has covered practically all fields of modern life, and basically, digital image processing which has been widely utilized in the medical field, including structure segmentation and image enhancement, have provided great support for diagnosis and better medical prognosis $[11,12]$.

The aim of this work is to propose new computational methods to carry out enhancement and segmentation of SARSCov-2 high-resolution microscopic images. Moreover, due to the importance of the obtained results, this first work will be addressed to application of the proposed algorithm. The proposed strategy obtained very satisfactory results, and we validated its performance, together with specialist physicians, on a set of 1005 images. A second paper will deeply analyze the theory related to these algorithms.

The rest of paper is organized as follows: in Section II, the materials and methods are given. Section III slightly outlines some theoretical and algorithmic aspects. A second paper will deepen into these issues. Section IV contains the obtained results and discussion. We describe our conclusions in Section $\mathrm{V}$.

\section{Materials and methods}

\subsection{Medical methodology}

Studied microscopic images were captured from nasopharyngeal swabs collected from Cuban individuals with COVID-19 symptomatic and RT-PCR positive for SARS-CoV- 
2. Samples were treated with an aldehyde solution and processed by Scanning Electron Microscopy, Confocal Microscopy and, Atomic Force Microscopy [13]. We were able to complete a database of more than 1010 microscopic images.

The structure identification of the SARS-CoV-2 virus is essential towards understanding its historical resemblance to other coronaviruses (mode of infection, mechanism of entry at tissue site of infection, and the replication process in the infected cells) [14]. Particularly, high-resolution microscopic studies are essential in identifying the etiological agent of several outbreaks. In the case of SARS-CoV-2, the Scanning Electron Microscopy (SEM) images provide fundamental data of the structural aspects of the virus and must be a guiding point in therapeutic development [13].
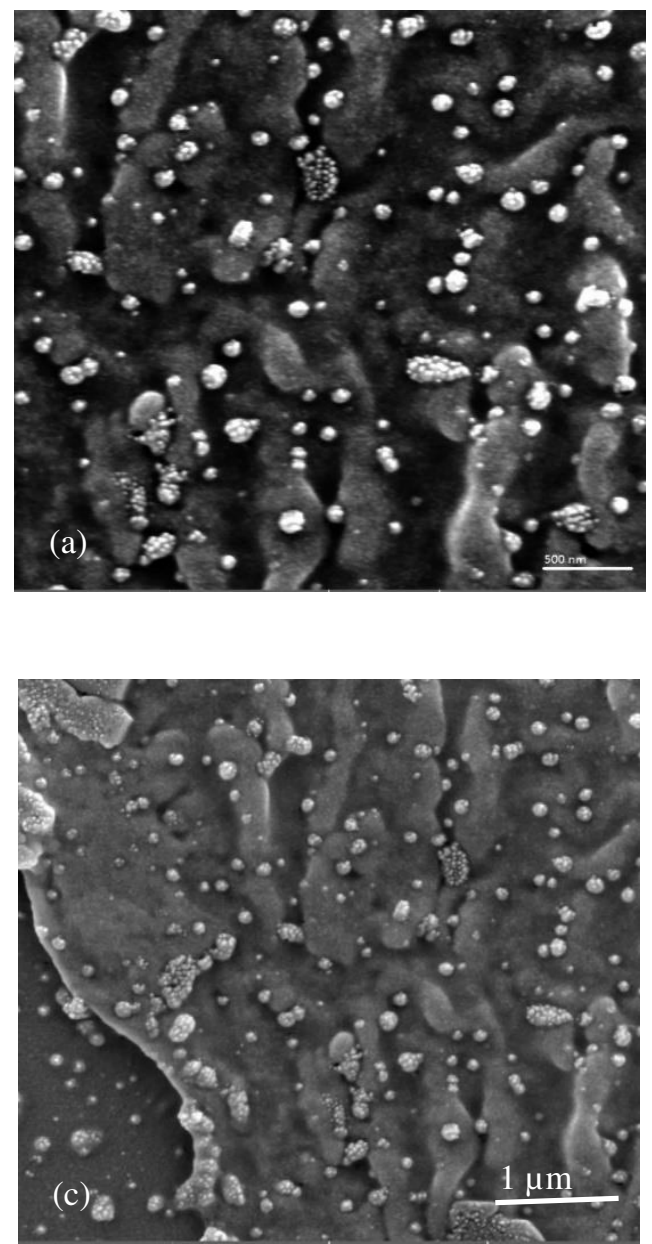

Fig 1. Characteristic of coronavirus microscopic images. a), (b), (c) and (d), original microscopic images.

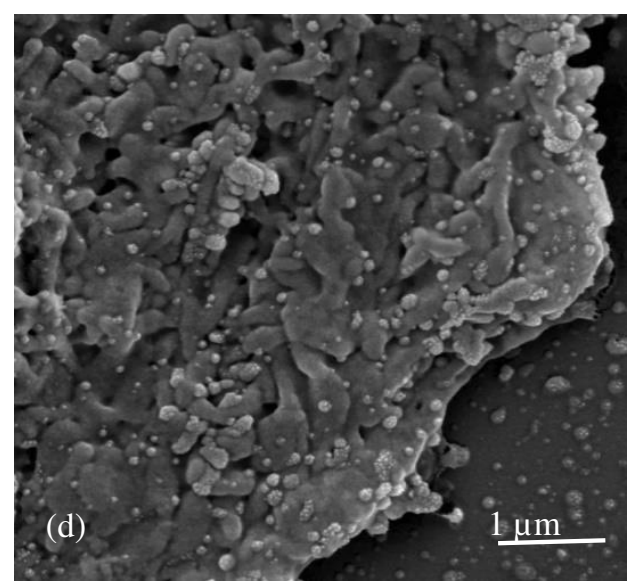

Clinical specimens were inactivated for 12 hours in a solution of $25 \%$ formaldehyde and $5 \%$ glutaraldehyde before microscopy study. Finally, we processed the inactivated samples by SEM. The coverslips were sputter-coated with gold (thickness of $10 \mathrm{~nm}$ ) and viewed under the MIRA3-TESCAN Scanning Electron Microscope at $10 \mathrm{kV}$ [13].

\subsection{Characteristics of studied images}

The dataset has more than 1010 images, but by space problem, we only give eight examples. Figure 1 shows typical coronavirus images, which we captured via the SEM. Figure 2 shows other examples.

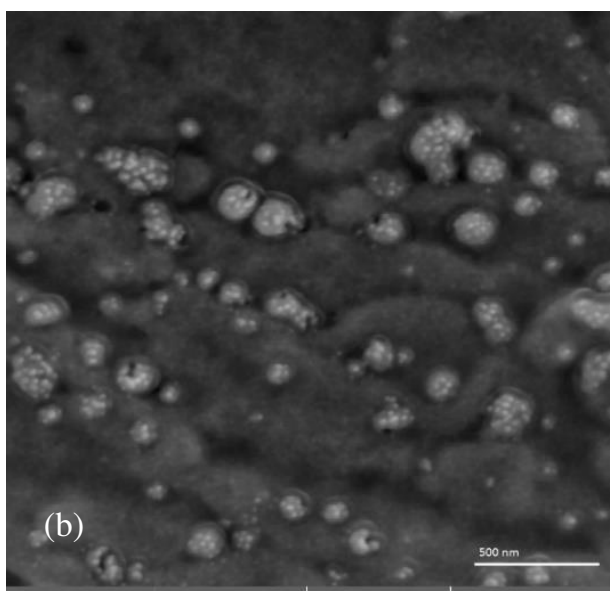



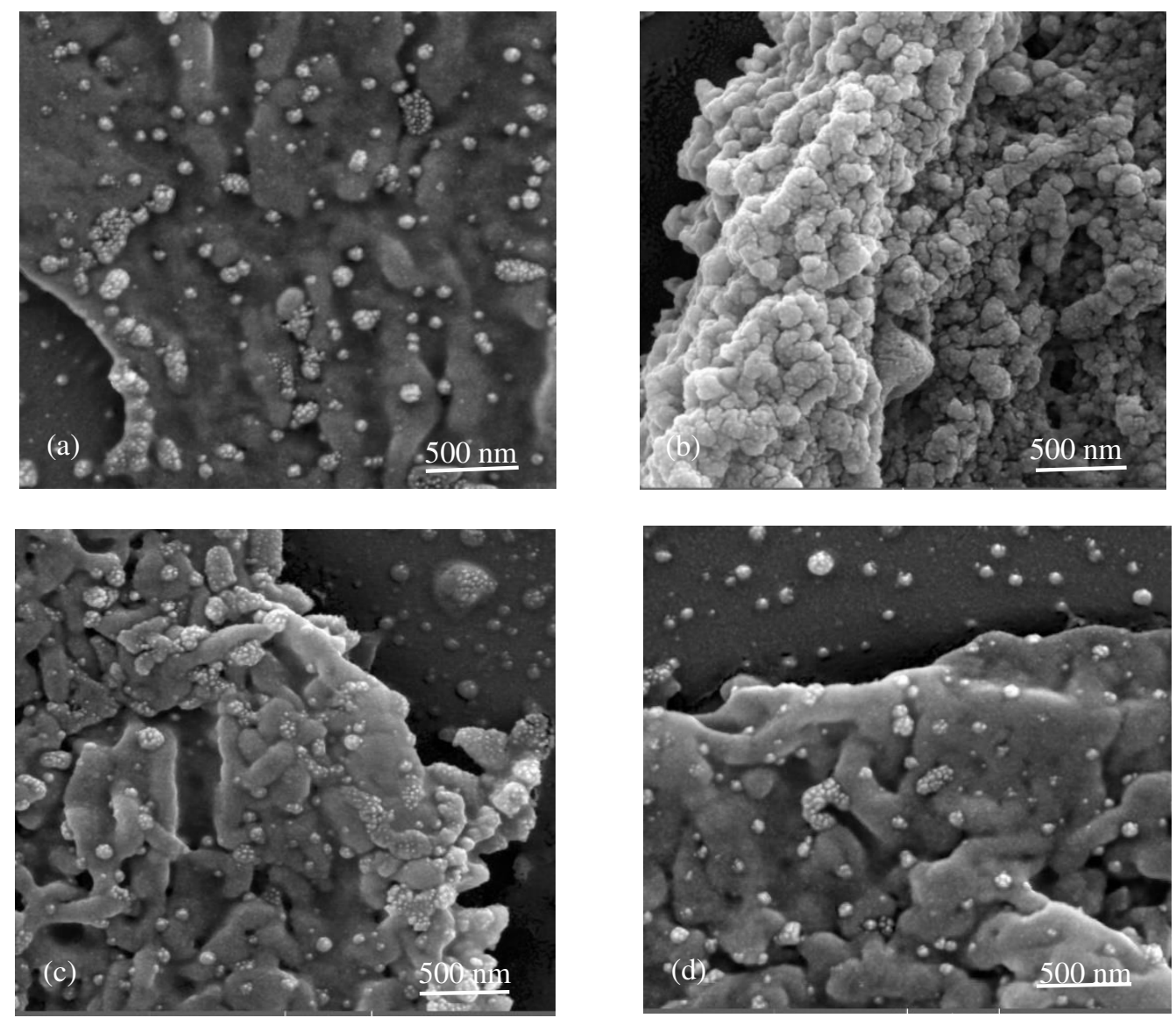

Fig 2. Characteristic of coronavirus microscopic images. (a), (b), (c) and (d), original microscopic images

In Figure 1 and 2, one can observe several notable characteristics of these microscopic images, which are common to typical microscopic images we encounter in the study of the novel coronavirus. For example, in Figure 3, one can see a horizontal profile through images of Figure 1 (b) and Figure 2 (d); this is a plot of the pixel intensities along a single row. One can note:

1. The intensities are slightly clearer within the virus than in the local surrounding background (Observe in Fig. 3, the " $y$ " axis). We pointed out that this observation holds only within the local surroundings.

2. One can observe high local variation of intensities both, within the coronavirus and within the background. However, the local variation of intensities are higher within the virus than in background regions (see Fig. 3).

3. It is possible to see (see Fig. 1 and Fig. 2) high variability of coronavirus in both, size and shape.

4. The contrast of intensities between the coronavirus and the background is low. This is evident from the intensities of histogram (see Fig. 4). This histogram is uni-modal, reflecting the low separation in average intensities of the virus and background
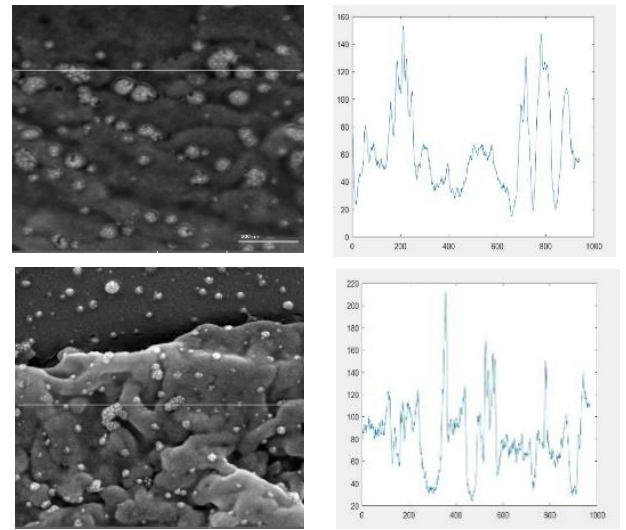

Fig. 3. An intensities profile through the images. We indicated the profile by a white line.
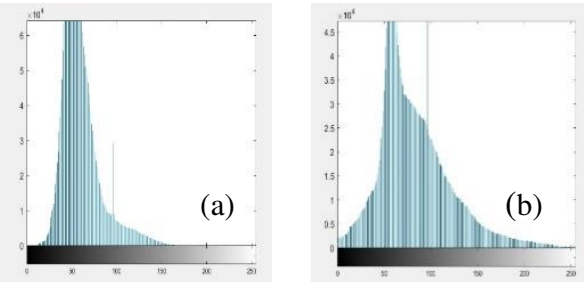

Fig. 4. Histograms. Fig. 4 (a), from Fig. 1 (b). Fig. 4 (b), from Fig. 2 (d). 
Due to the acquisition protocol and to the already mentioned physical phenomena, microphotographs are corrupted by a large amount of noise. One can see the ripples in the histograms and profiles (see Fig. 3 and Fig. 4). In addition, the observer can note, which draws noticeably attention, the certain blurring that exists in all these images. Across the entire image, changes in intensity levels cannot reliably distinguish $S$-spikes, due to possible non-uniformity of the average background intensity and the low contrast between virus and background. However, within a region of microphotographs, changes in intensity levels can effectively distinguish $S$-spikes, since locally a virus is clearer than its surrounding background. In other words, one can notice that coronavirus areas (the $S$-spikes) are hiper-dense regions.

The previous paragraph point out the need of working in the region of interest and locally. This strategy ensures that one taken into account the correlation among pixels, which is of great importance for getting good results, and addition, has a notable significance in the design of algorithms [15].

In Figures 5, we represent a $3 D$ projection of a region of interest (ROI) of Figures 1 (b) and 2 (d). It is possible see that the crests are smooth and peaks are not well distinguished. This is $3 D$ visualization of the blurring exists in these microphotographs.

The characterization of the study images is of supreme importance, and this should be a basic principle to have present. Many times, this determines the strategy to follow, and one should have in mind that the best result of analysis and interpretation of the image depends on segmentation quality [16].
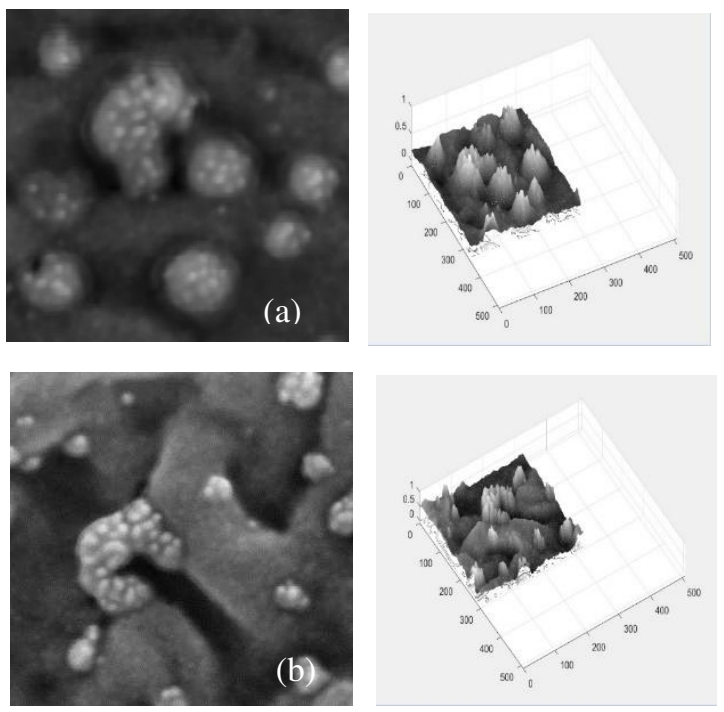

Fig. 5. (a), A ROI from Fig. 1 (b). (b), A ROI from Fig. 2 (d). Observe the blurring in the microphotographs, and the smooth of peaks.

\section{Some theoretical and algorithmic aspects}

In this section, due to the importance of the obtained results, we only outline lightly some theoretical and algorithmic aspects. In other words, we will address to application of the proposed algorithms. A second paper will deeply analyze the theory related to this algorithm.

\subsection{Some definitions}

Definition 1: (Regional maximum): A regional maximum at altitude $h$ of greyscale image $I$ is a connected component $C$ de $T_{h}(I)$ such that $C \cap T_{h+l}(f)=\varphi$.

We consider the successive thresholds $T_{h}(I)$ of $I$, for $h=0$ to $L$ 1 [16],

$$
T_{h}(I)=\left\{p \in D_{I} / I(p) \leq h\right\}
$$

They constitute the threshold decomposition of $I$, where these sets satisfy the following inclusion relationship:

$$
T_{h}(I) \subseteq T_{h-1}(I) \quad \forall h \in[0, L-1],
$$

where $L$ is the number of gray levels.

In Figure 6, one can observe a representation of regional maxima in a greyscale image. In this case, we considered the regional maximum as the $S$-spikes, where one can see that are justly the hiper-dense areas (see Fig. 1 and Fig. 2).

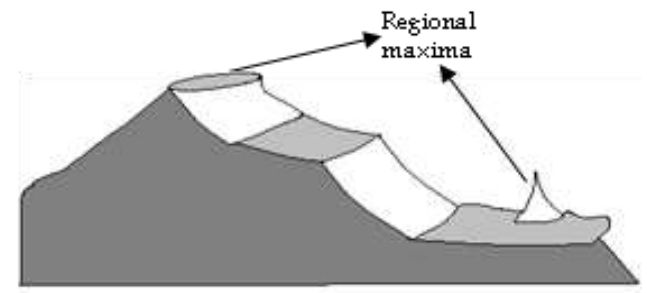

Fig. 6 Regional maxima in a greyscale image

Definition 2: (Grayscale reconstruction): The grayscale reconstruction $\rho_{I}(J)$ of $I$ from $J$ obtained by iterating grayscale dilations of $J$ "under" $I$ until stability is reached, that is,

$$
\rho_{I}(J)=\underset{n \geq 1}{\vee} \delta_{I}^{(n)}(J)
$$

Definition 3: The $h$-dome image $D_{h}(I)$ of the $h$-domes of a greyscale image $\boldsymbol{I}$ given by

$$
D_{h}(I)=I-\rho_{,}(I-h)
$$

The $h$-dome transformation extracts light structures without involving any size or shape criterion. The only parameter $(h)$ related to the height of these structures. In the case of coronavirus $S$-spikes segmentation, this parameter was of vital importance.

Definition 2: (Grayscale reconstruction): The grayscale reconstruction $\rho_{I}(J)$ of $I$ from $J$ obtained by iterating grayscale dilations of $J$ "under" I until stability is reached, that is,

$$
\rho_{I}(J)=\underset{n \geq 1}{\vee} \delta_{I}^{(n)}(J)
$$


Definition 3: The h-dome image $D_{h}(I)$ of the h-domes of a greyscale image $\boldsymbol{I}$ given by

$$
D_{h}(I)=I-\rho_{\boldsymbol{I}}(I-h)
$$

The h-dome transformation extracts light structures without involving any size or shape criterion. The only parameter $(h)$ related to the height of these structures. In the case of coronavirus $S$-spikes segmentation, this parameter was of vital importance.

\subsection{About of strategies}

All researches carried out with the proposed algorithm worked locally. This was the principal characteristic of designed algorithms for segmenting and enhancement the microphotographs of the novel coronavirus.

In effect, when we locally work with the algorithms, the spatial information is taken in consideration (and implicitly the correlation among the pixels), and we obtain a major stability in performance of strategies. One of the problems that have the classical threshold operators, it is to assume that the pixels are statistically independent [15].

\section{Algorithm No.1}

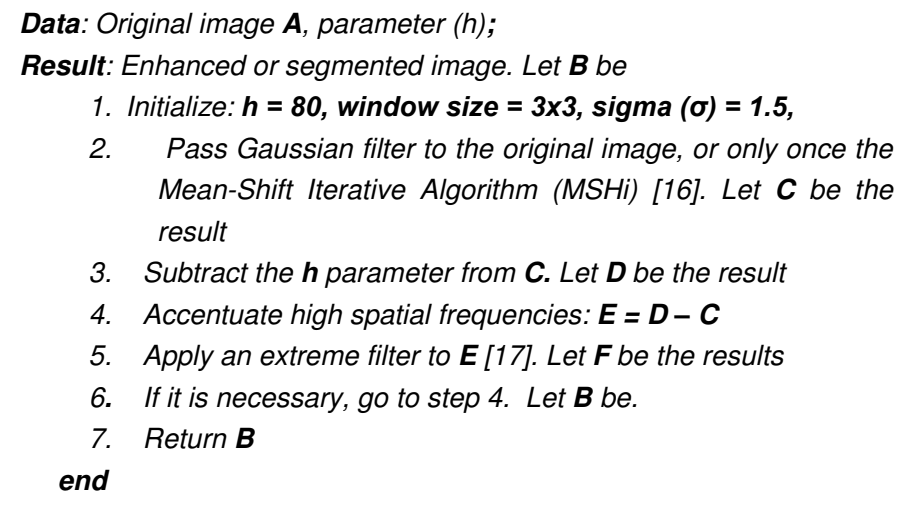

A deep explanation of the theory behind Algorithm No.1 and variant of it will appear in a second paper. Here, we will give a brief review of this.

The Gaussian filter was used to diminishing the noise in the original images. However, the MSHi algorithm has a performance more precise [16], but it consumes more computational time. In the case of Gaussian filter, the best performance was obtained using $\sigma=1.5$. The used window size was $3 \times 3$. A larger dimensional window caused a loss of information in the microphotographs.

The value of the $h$ parameter determines the processing characteristics. For a small value of $h$, the $S$-spikes were improved (more contrasted) in relation to the original image. While, for a large value of $h$, the $S$-spikes were isolated (segmented), where the S-spikes appeared clear on a black background. The importance of improving or segmenting the $S$ spikes is due to those areas that showed an S-spike high density and pseudohyphae projections appeared to be related to an active zone of viral germination [13].

\section{Experimental results. Discussion}

In computer vision, image enhancement and particularly segmentation, i.e., classification of the image gray-level values into homogeneous areas are recognized as ones of the most important steps in any image analysis system.

In Figure 7, we show a first example of the obtained result of carrying out the Algorithm No.l for different values of $h$ parameter, and applied to the image of Figure 1 (a).

In Figure 7, one can see that the processed images of applying the Algorithm No. 1 were all enhanced; that is, contrast increased, and the $S$-spikes are evident and virion particles shows the urchin-shape. Note that for small values of $h$, we obtained a better-contrasted image. However, as the value of $h$ increased, the S-spikes begin to segment, where one can see white areas on a black background, and in all images, it is possible to observe the edges more defined. On the other hand, with the images (e) and (f), we can to carry out an automatic analysis of the density of S-spikes (number of S-spikes/ area unit); that is, automatic count of white points on a black background area.

When one analysis the profiles of processed images, the difference with those that appear in Figure 3 is remarkable. In effect, observe in Figure 8 that the peaks of the profiles, for different $h$ values, begin to flattening. In other words, parallel lines to the " $x "$ axis on peaks are indicative of equal intensity levels (segmented zone). In addition, the peaks begin to separate, which shows the isolation of the S-spikes in the different areas where the coronavirus are. The intensity of background reached zero value.

Moreover, in Figure 7, the following question is present: $¿$ Which image to choose or which to select as the best processed image? The answer to this question is not direct, because it depends on the aim of observer or from the application itself; that is, segmentation is heavily dependent on the application [18]. A theoretical analysis of the $h$ parameter and its influence will appear in an upcoming publication. Figure 9 shows the obtained result of processing the image of Figure 1 (b) for three values of $h$ parameter.

Note that the algorithm has very similar behavior to the image in Figure 7 (a), which shows the stability of this strategy. In effect, for small values of the $h$ parameter, we achieved a contrast enhancement, while that for high values, we obtained a segmented image. Values of the $h$ parameter in the range 10 to 59, for the purposes of this application, we considered it small. Superiors to this interval, we considered them high. On the other hand, it is evident that the algorithm in all cases improved contrast and accentuated the high spatial frequencies (edges and the S-spikes). See the original microphotograph (very blurry) with relation to processed images. In a $3 D$ representation, one can better see this claim (see Fig. 10). 
In Figure 10 (a), observer can better appreciate the degree of blurring in the original image, where the S-spikes seem to be out-of-focus. Note in the $3 D$ representation that the crests are flattened (see image 10 (b)). However, in Figure 10 (c), one observes the $S$-spikes and the crown-shape is evident in some viral particles, resembling the typical morphology of the coronaviruses [13]. The image has better contrast and edges more defined. The $3 D$ representation of this processed image shows acute, separated crests and high peaks.

Other processing examples carried out with the Algorithm No. 1 , for two $h$ parameter values, appear in Figures 11, where one can note the stability of the Algorithm. No. 1 for different images. See that both, in the enhanced and segmented images the $S$-spikes are easy to distinguish. However, one cannot say the same in original images, where the $S$-spikes appear very blurred. We carried out more than 210 experiments on different images and we obtained the same results.
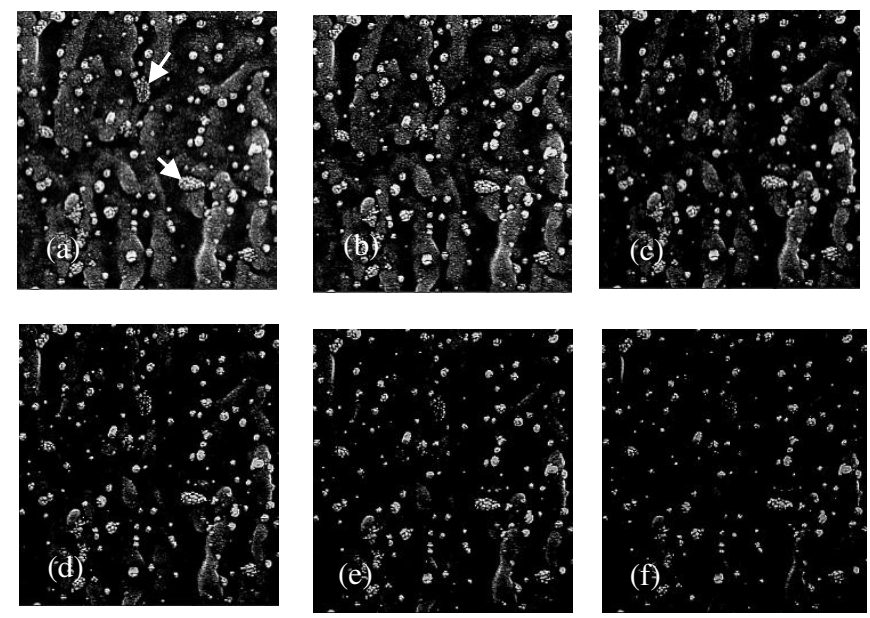

Fig. 7. Application of the Algorithm No. 1 to the imagen of Fig. 1 (a) at different values of h parameter. (a) $h=15$, (b) $h=30$, (c) $h=60$, (d) $h=80$, (e) $h=100$, (f) $h=140$. In (a), the arrows show white dots, which representing the $S$-spikes.
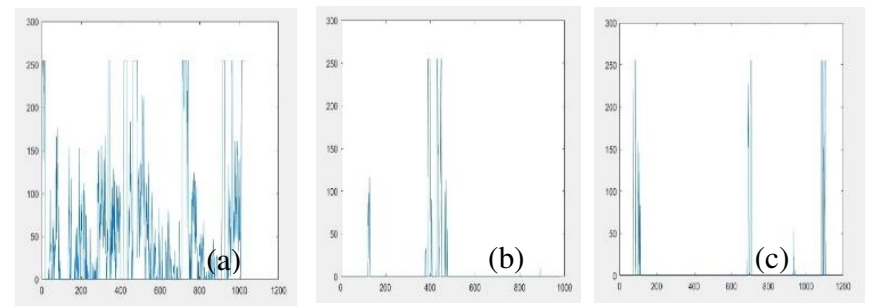

Fig.8. An intensity profile through a processed image from Fig. 3. (a) $h=15$, (b) $h=80$, (c) $h=140$. Observe that the peaks of profiles begin to flattening (see profiles of Fig. 3).
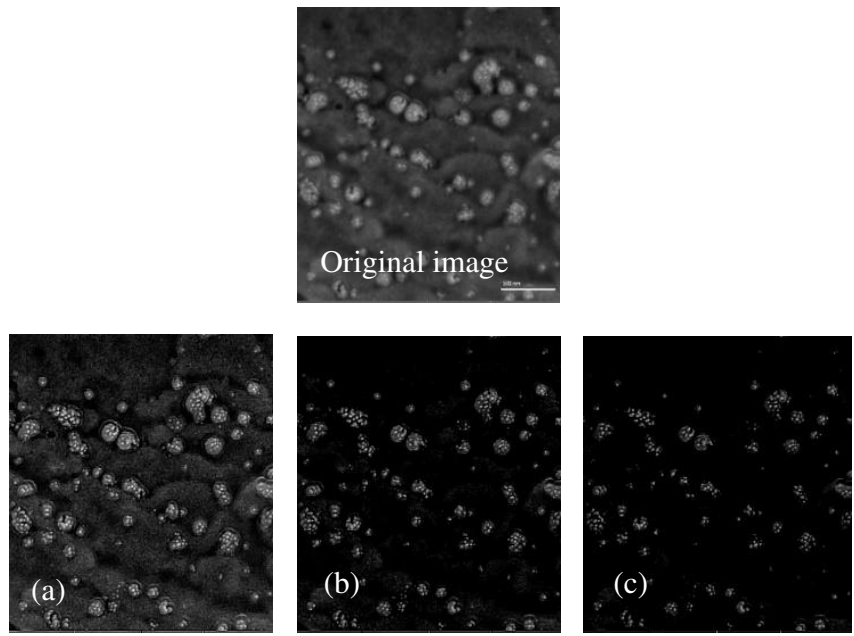

Fig. 9. Application of the Algorithm No. 1 to imagen of Fig. 1 (b) for three values of $h$ parameter. (a) $h=15$, (b) $h=60$, (c) $h=80$. We show the original image for a better visual comparison.
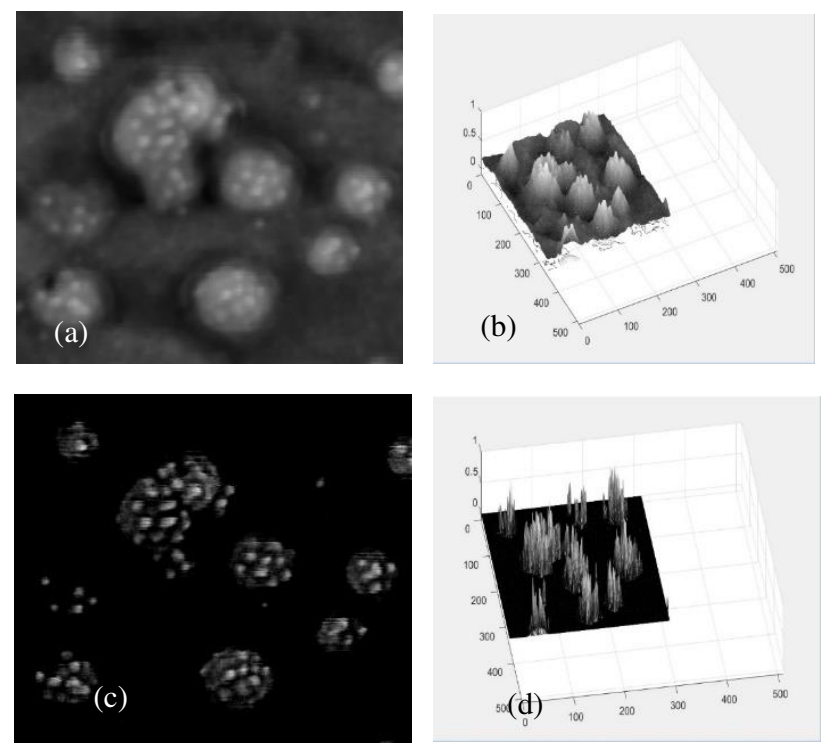

Fig. 10. A region of interest (ROI) from the image in Figure 1 (b). (a) Original ROI, (b) $3 D$ representation of ROI, (c) Processed image, (d) $3 D$ representation of processed ROI.

\subsection{Comparisons with other methods}

In this section, we will compare our strategy with two recognized classical techniques. Namely, a histogram equalization (contrast improvement method) and the Otsu's method (segmentation). Such comparison is necessarily incomplete, as we could only be a comprehensive comparison in a survey paper, given the information volume existing in the literature. Instead, we selected two recognized classical techniques, which are suitable for the kind of used images in our study. In Figure 12, we show the obtained results with the histogram equalization and the Otsu's method, and our algorithm. 
In a visual comparison together with specialist physicians (more than 10), one can notice that the histogram equalization was not able to discriminate the $S$-spikes; that is, happened quite the opposite; the method joined the $S$-spikes and produced an over-saturation. Something similar happened with the Otsu's method. However, our algorithm accentuated and isolated the $S$-spikes. The theoretical explanation of why this will appear in a future publication.
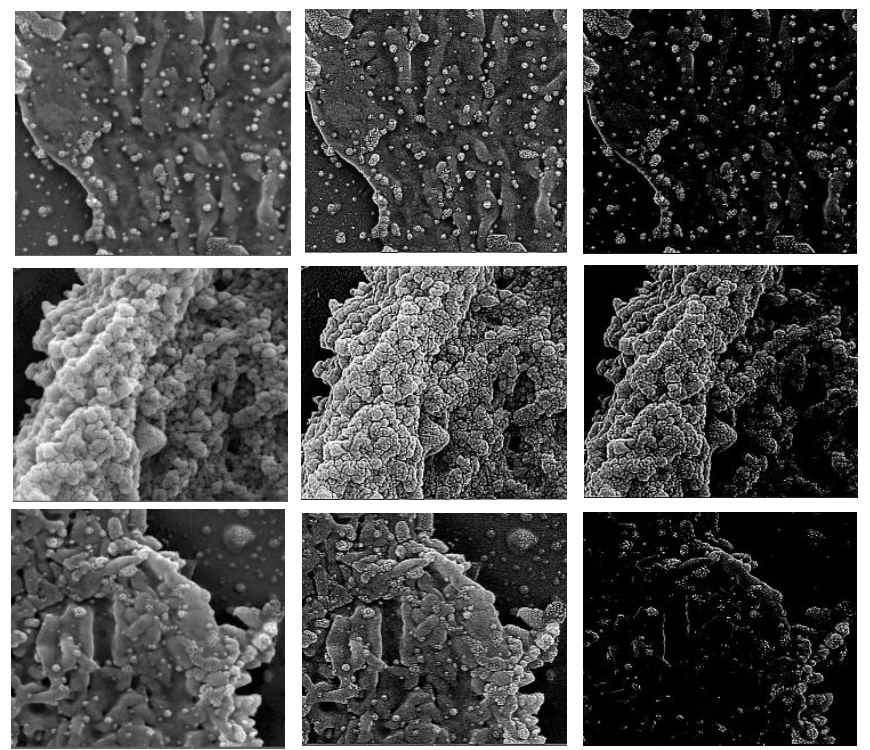

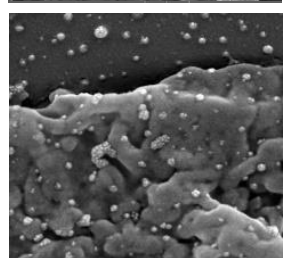

(a)

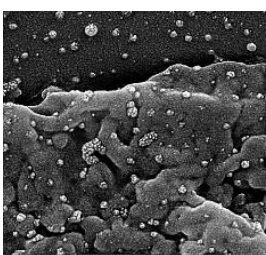

(b)

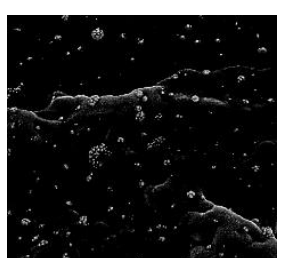

(c)
Fig. 11. Enhanced and segmented images applying the Algorithm No. 1. (a) Original images, (b) $h=15$, (c) $h=85$.
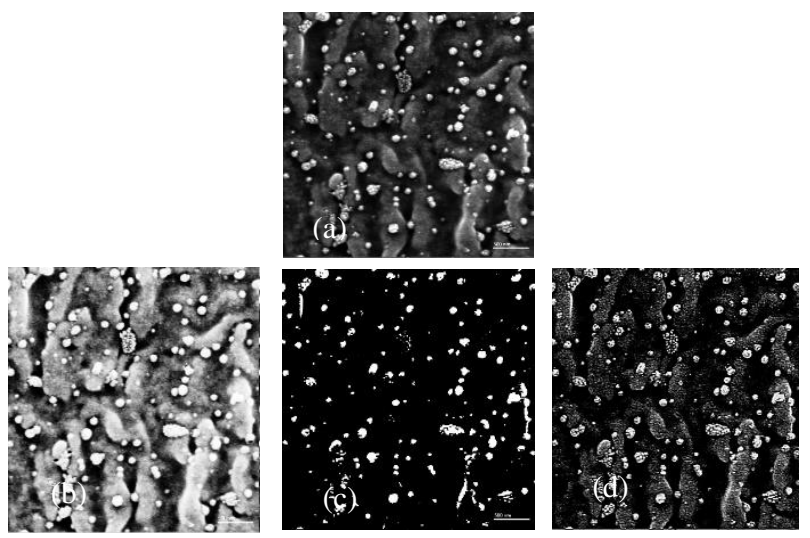

Fig. 12. Comparisons with two classical methods. (a) Original image, (b) Histogram equalization, (c) Otsu's method, (d) Our algorithm. It is evident the obtained result with our strategy
Other examples of comparisons with other images appear in Figures 13 and 14.
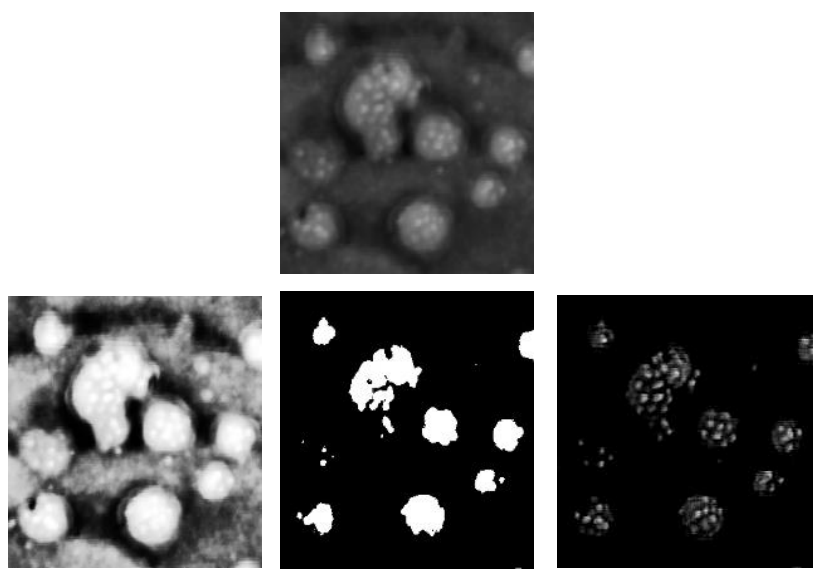

Fig. 13. (Iden. to Fig. 12). However, in this case, the histogram equalization and the Otsu's method joined more the S-spikes and produced more over-saturation. Observe the blurring of original image. Our strategy always performed well.

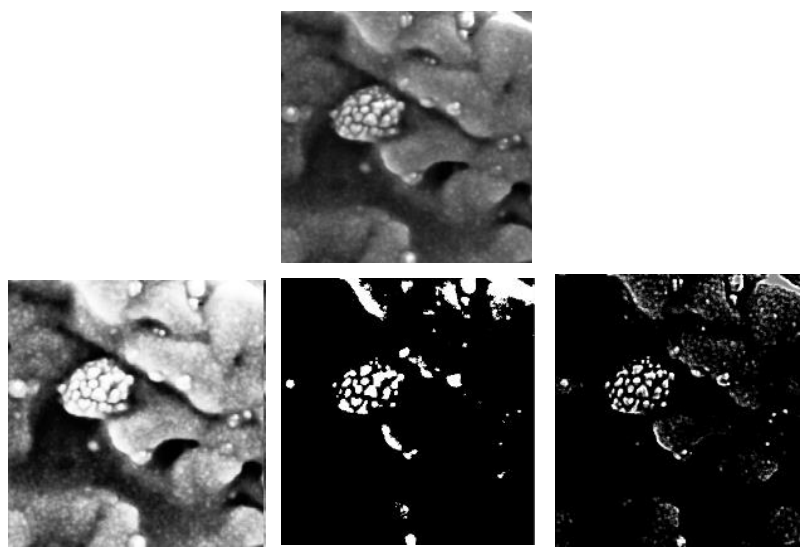

Fig. 14. (Iden. to Fig. 12). In this case, the classic methods performed better, although in both, a bit of over- saturation there was. Notice that the original image is less blurred. Our algorithm worked well in both situations

\section{Conclusions}

We presented a strategy for enhancement and segmentation of microscopic images of the novel coronavirus. The basic idea was to take advantage of the characteristics of microscopic images of the novel coronavirus (hyper-dense zones) for the creation of an algorithm, with a minimum of parameters, which would be able to improve and segment the S-spikes. In this sense, we proposed a variant of the $h$-dome transformation that consisted in subtracting an $h$ parameter to separate hyper-dense areas and work locally with high spatial frequencies. The proposed algorithm showed accuracy and had good performance and stability in the enhancement and isolation of S-spikes of the SARS-CoV-2 coronavirus. Finally, we compared our algorithm with two well-established classical methods, and proved that, in all cases, it was superior in the 
processing of microphotographs. A next publication will carry out an algorithmic and theoretical analysis of the results here obtained.

\section{References}

1. $\mathrm{Yu}$ Chen, Qianyun Liu, and Deyin Guo: "Emerging coronaviruses: genome structure, replication, and pathogenesis", Journal of medical virology, 92 (4):418-423, 2020.

2. Catharine I Paules, Hilary D Marston, and Anthony S Fauci: "Coronavirus infections|more than just the common cold", Jama, 323 (8):707-708, 2020.

3. Anwaar Ulhaq, Asim Khan, Douglas Gomes and Manoranjan Paul: "Computer Vision for COVID-19 Control: A survey”, ArXiv:2004.09420v2 [eess.IV] 5 May 2020.

4. Ophir Gozes, Ma'ayan Frid-Adar, Hayit Greenspan, Patrick D. Browning, Huangqi Zhang, Wenbin Ji, Adam Bernheim, and Eliot Siegel: "Rapid AI Development Cycle for the Coronavirus (COVID-19) Pandemic: Initial Results for Automated Detection \& Patient Monitoring using Deep Learning CT Image Analysis", This work has been submitted to Radiology: Artificial Intelligence for possible publication,

https://www.researchgate.net/publication/339873521

5. Qingsen Yan, Bo Wang, Dong Gong, Chuan Luo, Wei Zhao, Jianhu Shen, Qinfeng Shi, Shuo Jin, Liang Zhang and Zheng You: "COVID-19 Chest CT Image Segmentation: A Deep Convolutional Neural Network Solution”, ArXiv:2004.10987v2 [eess.IV] 26 Apr 2020.

6. Deng-Ping Fan, Tao Zhou, Ge-Peng Ji, Yi Zhou, Geng Chen, Huazhu Fu, Jianbing Shen, and Ling Shao: "Inf-Net: Automatic COVID-19 Lung Infection Segmentation from CT Scans", https://doi.org/10.1101/2020.04.22.20074948

7. A. Amyar, R. Modzelewski and S. Ruan: "Multi-task deep learning based CT imaging analysis for COVID-19: Classification and Segmentation", https://doi.org/10.1101/2020.04.16.20064709

8. Bhupender S. Chhikara, Brijesh Rathi, Jyoti Singh and Poonam: "Corona virus SARS-CoV-2 disease COVID-19: Infection, prevention and clinical advances of the prospective chemical drug therapeutics", Chemical Biology Letters, 7 (1), 63-72, 2020.

9. Fred Plapp: "The COVID-19 Pandemic: A Summary", A Special Supplement from the Pathologist, 2020. www.thepathologist.com

10. Na Zhu, Dingyu Zhang, Wenling Wang, Xingwang Li, Bo Yang, Jingdong Song, Xiang Zhao, Baoying Huang, Weifeng Shi, Roujian Lu, Peihua Niu, Faxian Zhan, Xuejun Ma, Dayan Wang, Wenbo Xu, Guizhen Wu, George F. Gao,
D.Phil. and Wenjie Tan: "A Novel Coronavirus from Patients with Pneumonia in China, 2019", The new England Journal of Medicine, 382, 727-33, 2020. DOI: 10.1056/NEJMoa2001017

11. Gharbi, Michaël, Chen J, Barron J T, et al.: “Deep Bilateral Learning for Real-Time Image Enhancement". Acm Transactions on Graphics, 36 (4):118, 2017

12. Liu X, Guo S, Yang B, et al.: "Automatic Organ Segmentation for CT Scans Based on Super-Pixel and Convolutional Neural Networks", Journal of Digital Imaging, 31 (6), 2018.

13. Brian Mondeja, Odalys Valdes, Sonia Resik, Ananayla Vizcaino, Emilio Acosta, Adelmo Montalván, Amira Paez, Mayra Mune, Roberto Rodríguez, Juan Valdés, Guelsys Gonzalez, Daisy Sanchez, Viviana Falcón, Yorexis González, Vivian Kourí, Angelina Díaz and Maria Guzmán.: "SARS-CoV-2: High-Resolution Microscopy Study in Human Nasopharyngeal Samples", sent to Virology Journal, 2020. DOI: 10.21203/rs.3.rs-36154/v1

14. Chu H, Chan JF-W, Yuen TT-T, Shuai H, Yuan S, Wang Y, et al.: "Comparative tropism, replication kinetics, and cell damage profiling of SARS-CoV-2 and SARS-CoV with implications for clinical manifestations, transmissibility, and laboratory studies of COVID-19: an observational study", The Lancet Microbe. May. 1 (1):e14-23, 2020.

15. Rodríguez, R., Garcés, Y., Torres, E., Sossa, H. and Tovar, R.: "A vision from a physical point of view and the information theory on the image segmentation", Journal of Intelligent \& Fuzzy Systems, vol. 37, pp. 2835-2845, IOS Press, 2019. DOI: 10.3233/JIFS-190030.

16. Rodríguez, R., and Sossa, J. H.: Mathematical Techniques for Biomedical Image Segmentation, In R. Narayan (Ed.), Encyclopedia of Biomedical Engineering, vol. 3, pp. 64-78. Elsevier. ISBN: 9780128048290, Elsevier Inc. All rights reserved. Elsevier, Copyright (C) 2019.

17. Roberto Rodríguez and Juan H. Sossa, (In Spanish), Procesamiento y Análisis Digital de Imágenes, Book Published by "Ra-Ma®" Editorial, ISBN: 978-84-9964007-8, Printed in Spain, May 2011.

http://www.rama.es/busqueda/listaLibros.php?tipoBus=autor\&palabrasB usqueda $=$ Roberto+Rodriguez $\% 2 \mathrm{C}+\mathrm{Juan}+\mathrm{H} .+$ Sossa

18. Roberto Rodríguez, Didier Domínguez, Esley Torres and Juan H. Sossa, Image Segmentation through an Iterative Algorithm of the Mean Shift, Chapter of the Book entitled: "Advances in Image Segmentation", ISBN 978-953-510817-7, edited by Pei-Gee Peter Ho, Publisher: InTech, 2012 http://www.intechopen.com/books/advances-in-imagesegmentation/image-segmentation-through-an-iterativealgorithm-of-the-mean-shift 


\section{Figures}
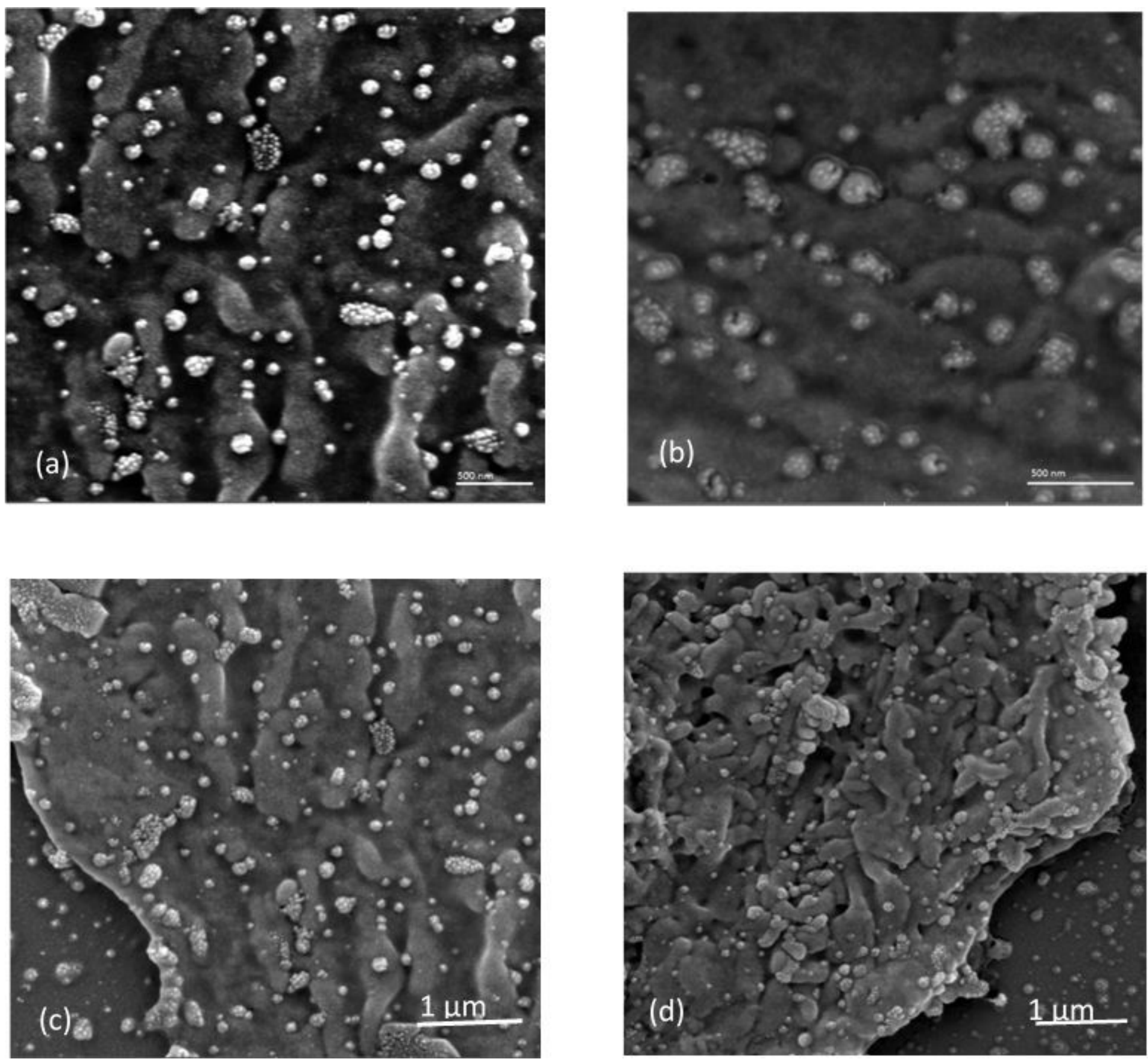

Figure 1

Characteristic of coronavirus microscopic images. a), (b), (c) and (d), original microscopic images. 

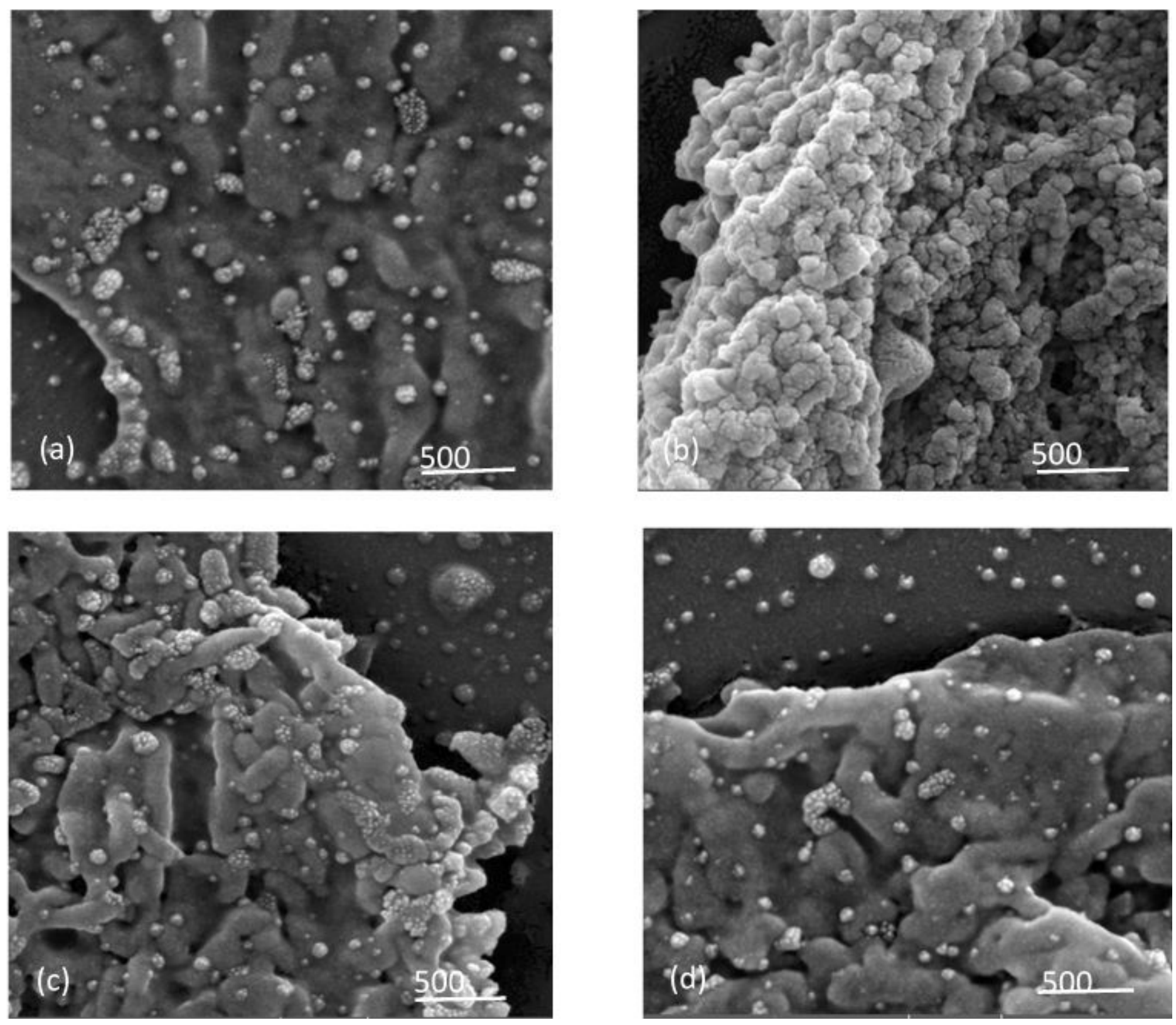

Figure 2

Characteristic of coronavirus microscopic images. (a), (b), (c) and (d), original microscopic images 

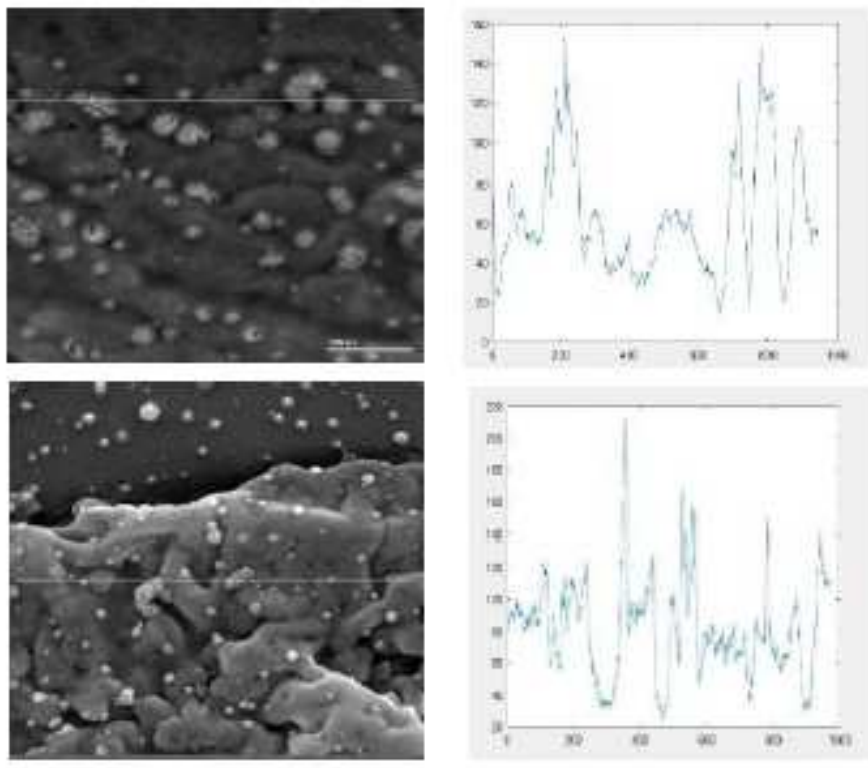

Figure 3

An intensities profile through the images. We indicated the profile by a white line.
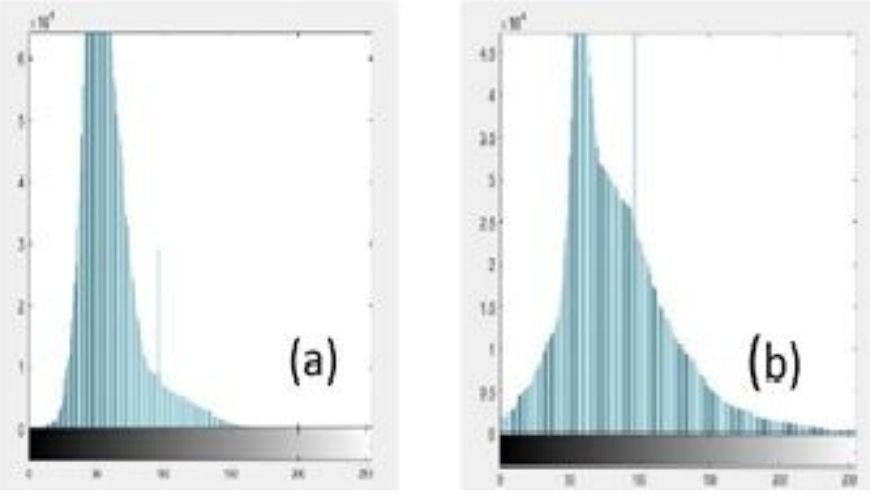

Figure 4

Histograms. Fig. 4 (a), from Fig. 1 (b). Fig. 4 (b), from Fig. 2 (d). 

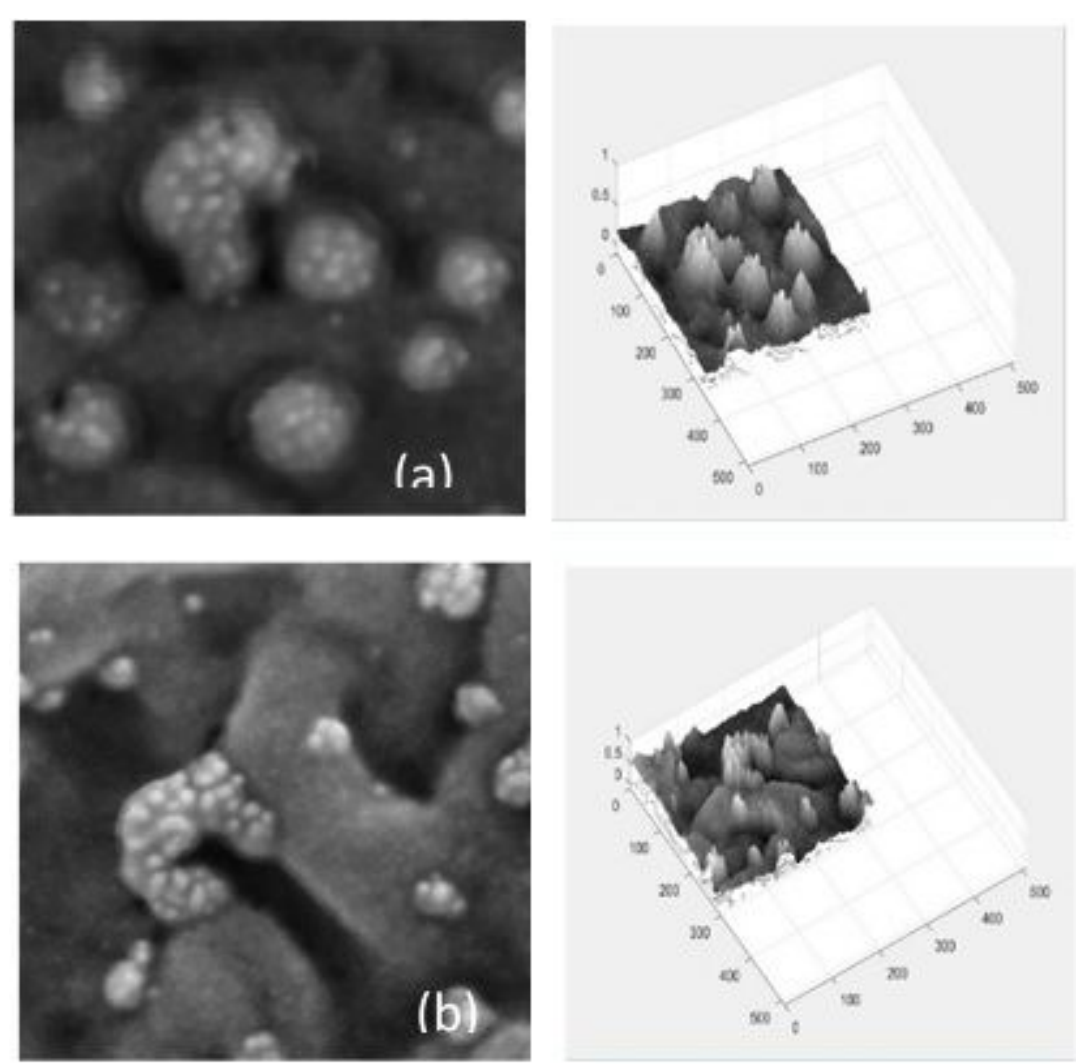

\section{Figure 5}

(a), A ROI from Fig. 1 (b). (b), A ROI from Fig. 2 (d). Observe the blurring in the microphotographs, and the smooth of peaks.

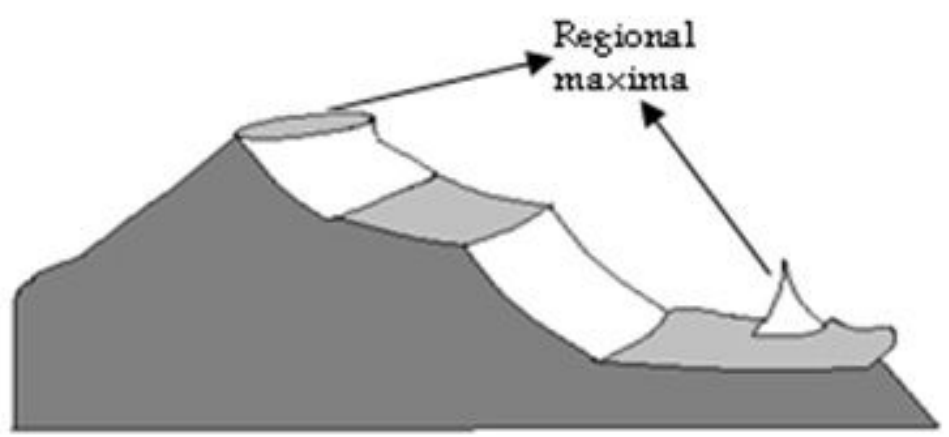

Fig. 6 Regional maxima in a greyscale image

Figure 6

Regional maxima in a greyscale image 

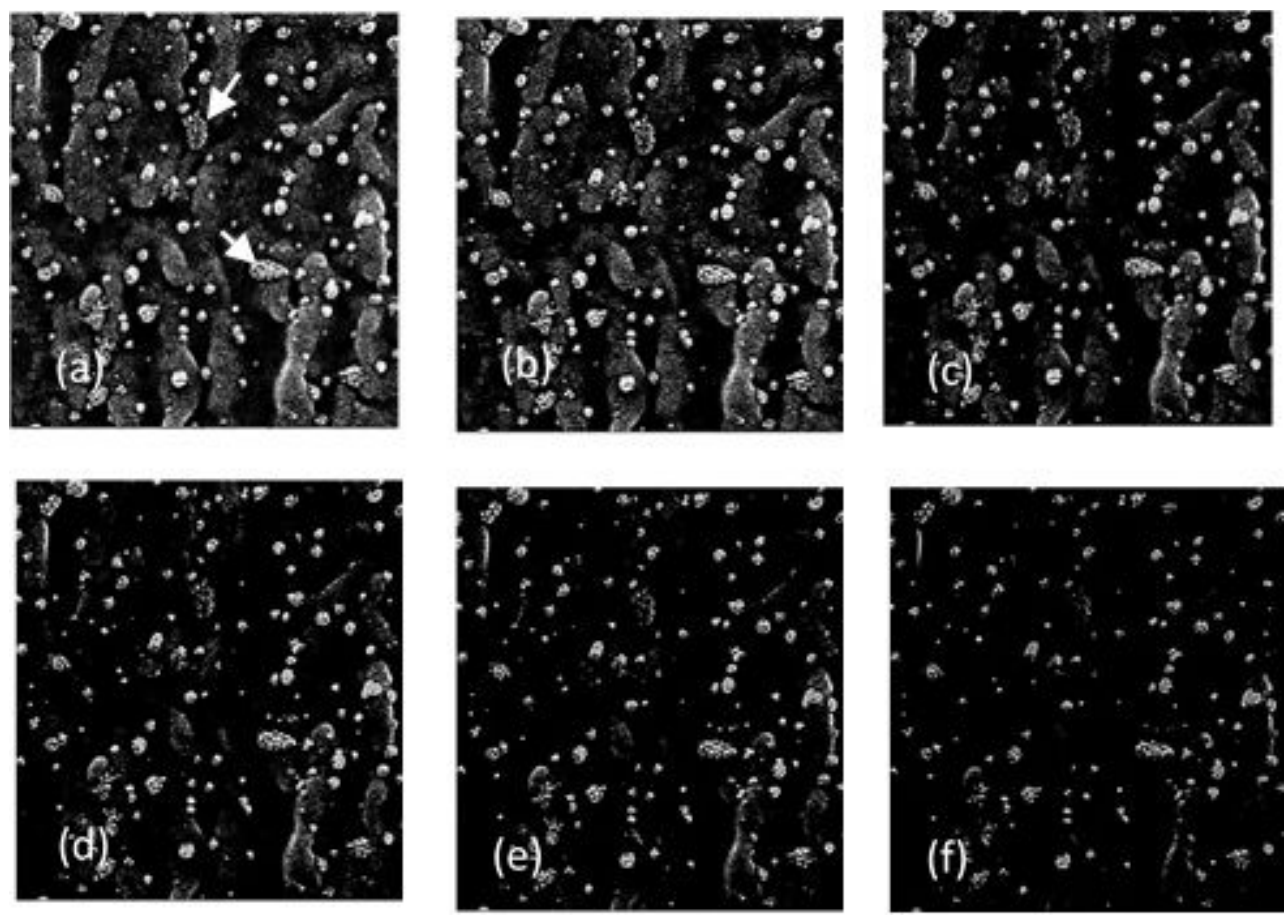

\section{Figure 7}

Application of the Algorithm No. 1 to the imagen of Fig. 1 (a) at different values of $h$ parameter. (a) $h=15$, (b) $h=30,(c) h=60,(d) h=80,(e) h=100,(f) h=140$. In (a), the arrows show white dots, which representing the S-spikes.
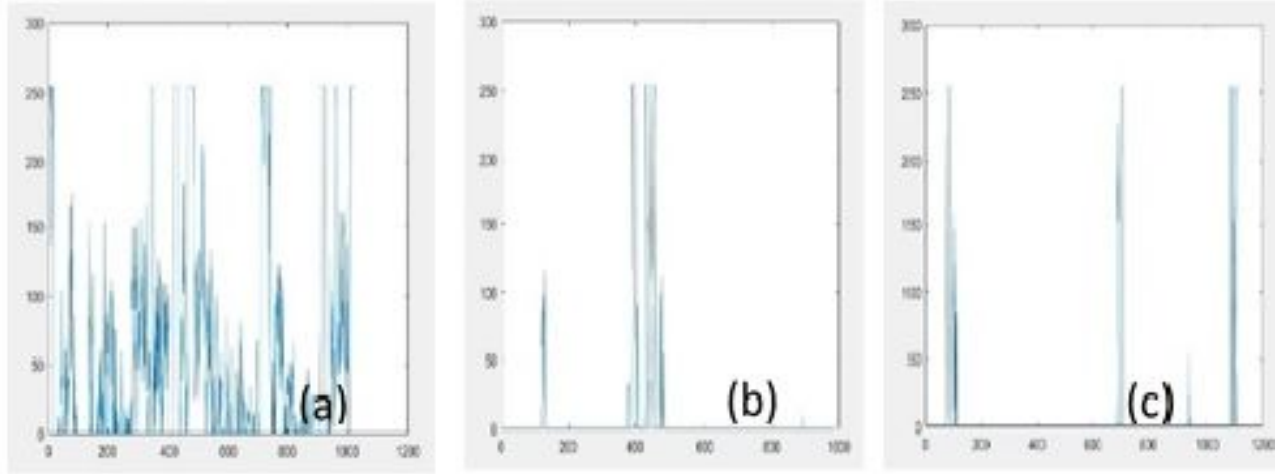

Figure 8

An intensity profile through a processed image from Fig. 3. (a) $h=15$, (b) $h=80$, (c) $h=140$. Observe that the peaks of profiles begin to flattening (see profiles of Fig. 3). 

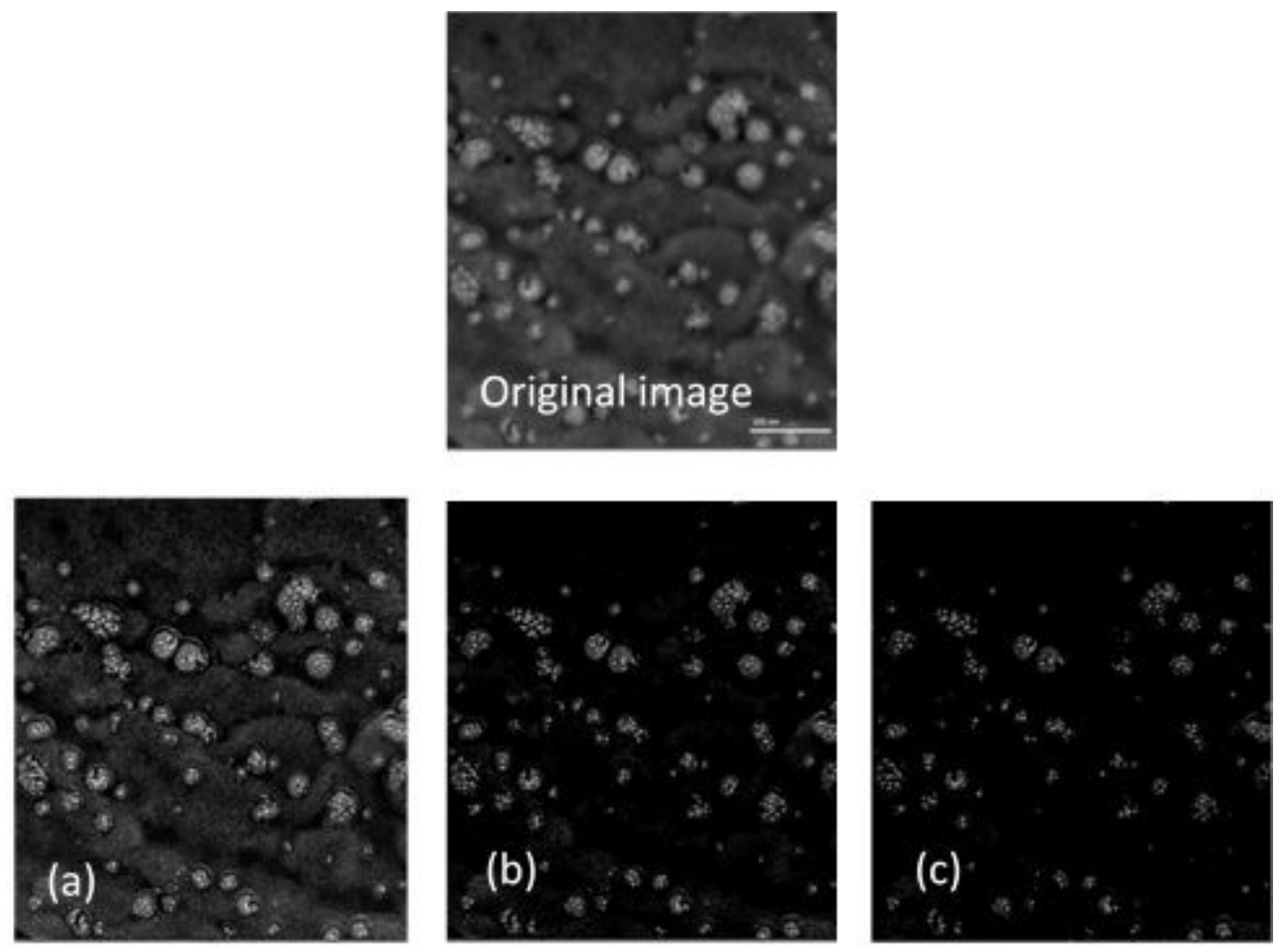

\section{Figure 9}

Application of the Algorithm No. 1 to imagen of Fig. 1 (b) for three values of h parameter. (a) $h=15$, (b) $h=60$, (c) $h=80$. We show the original image for a better visual comparison.
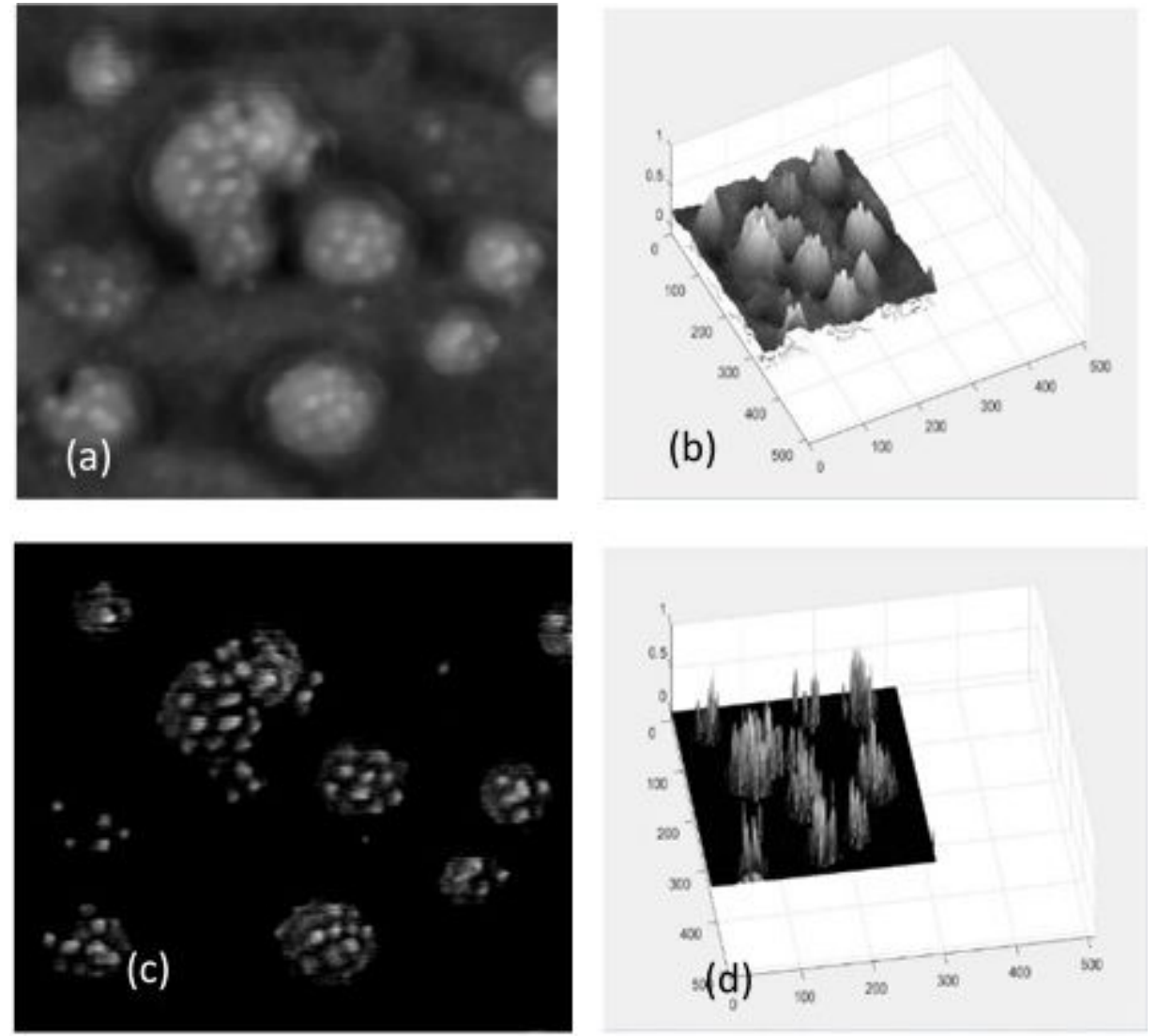

Figure 10 
A region of interest (ROI) from the image in Figure 1 (b). (a) Original ROI, (b) 3D representation of ROI, (c) Processed image, (d) 3D representation of processed ROI.
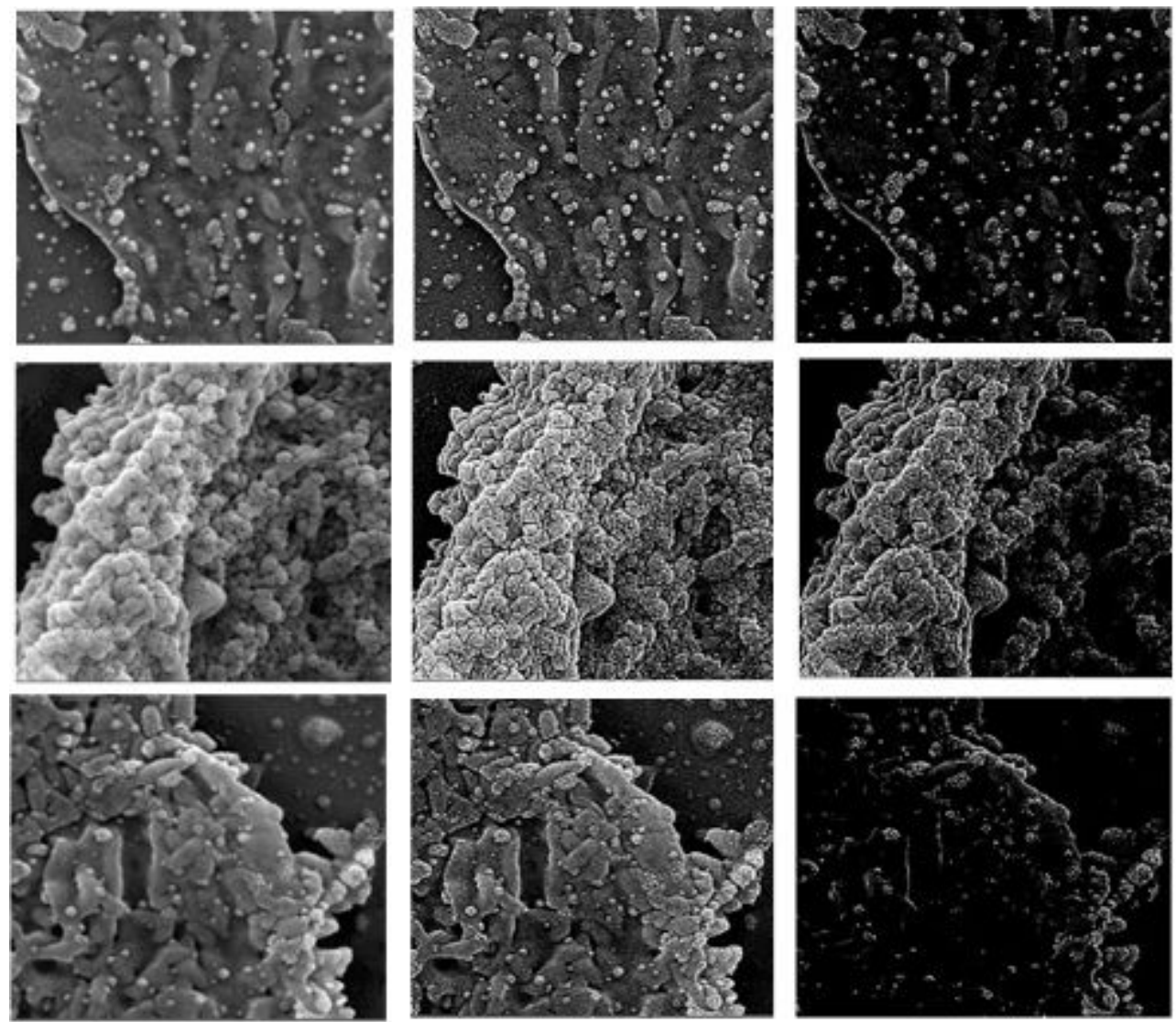

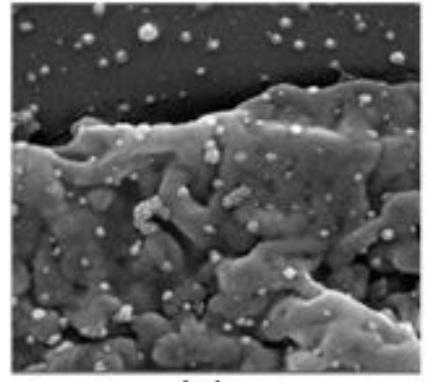

(a)

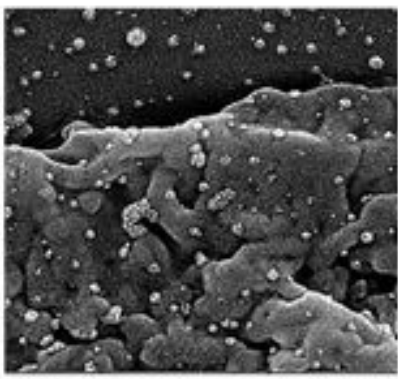

(b)

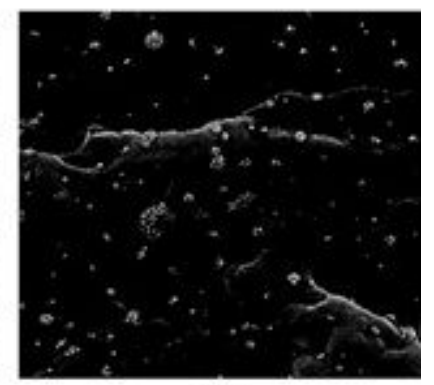

(c)

\section{Figure 11}

Enhanced and segmented images applying the Algorithm No. 1. (a) Original images, (b) $h=15$, (c) $h=85$. 

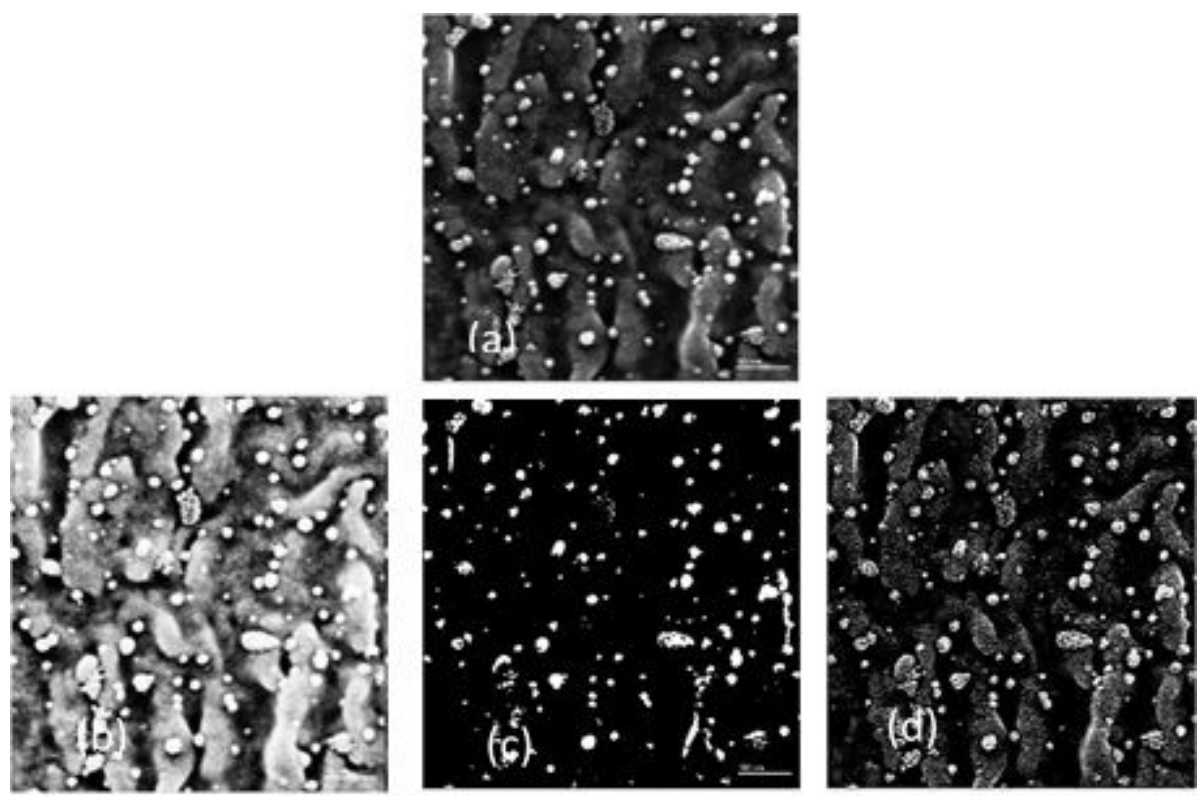

Figure 12

Comparisons with two classical methods. (a) Original image, (b) Histogram equalization, (c) Otsu's method, (d) Our algorithm. It is evident the obtained result with our strategy
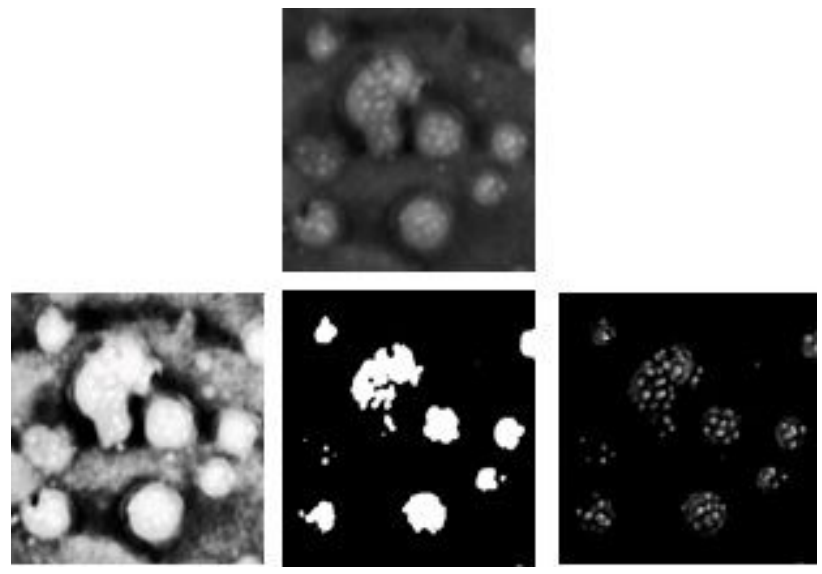

Figure 13

(Iden. to Fig. 12). However, in this case, the histogram equalization and the Otsu's method joined more the S-spikes and produced more over-saturation. Observe the blurring of original image. Our strategy always performed well. 


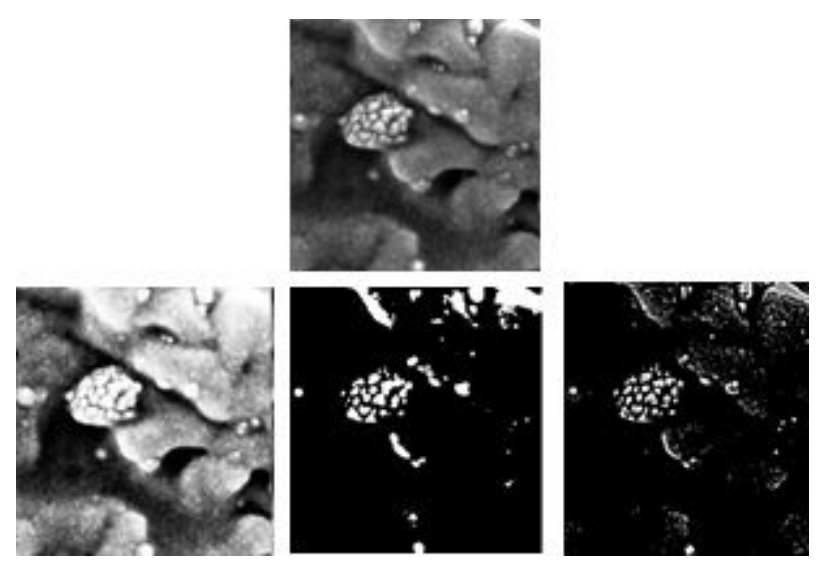

Figure 14

(Iden. to Fig. 12). In this case, the classic methods performed better, although in both, a bit of oversaturation there was. Notice that the original image is less blurred. Our algorithm worked well in both situations 